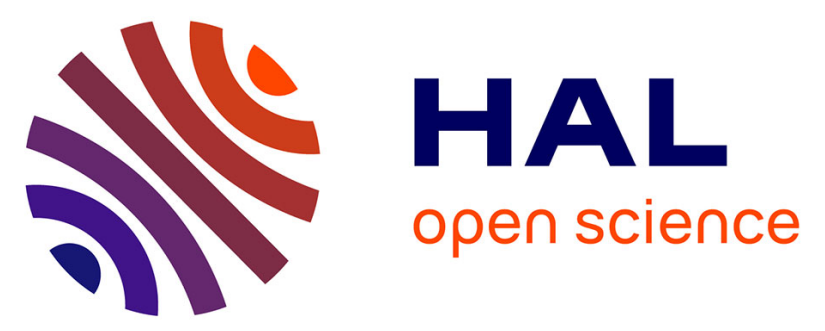

\title{
Combined Chemoradiation Therapy With Twice-Weekly Gemcitabine and Cisplatin for Organ Preservation in Muscle-Invasive Bladder Cancer: Long-Term Results of a Phase 1 Trial.
}

David Azria, Olivier Riou, Xavier Rebillard, Simon Thezenas, Rodolphe Thuret, Pascal Fenoglietto, Damien Pouessel, Stephane Culine

\section{To cite this version:}

David Azria, Olivier Riou, Xavier Rebillard, Simon Thezenas, Rodolphe Thuret, et al.. Combined Chemoradiation Therapy With Twice-Weekly Gemcitabine and Cisplatin for Organ Preservation in Muscle-Invasive Bladder Cancer: Long-Term Results of a Phase 1 Trial.: Conservative bladder cancer treatment. International Journal of Radiation Oncology, Biology, Physics, 2014, 88 (4), pp.853-9. 10.1016/j.ijrobp.2013.11.016 . inserm-00924350

\section{HAL Id: inserm-00924350 https://www.hal.inserm.fr/inserm-00924350}

Submitted on 6 Jan 2014

HAL is a multi-disciplinary open access archive for the deposit and dissemination of scientific research documents, whether they are published or not. The documents may come from teaching and research institutions in France or abroad, or from public or private research centers.
L'archive ouverte pluridisciplinaire HAL, est destinée au dépôt et à la diffusion de documents scientifiques de niveau recherche, publiés ou non, émanant des établissements d'enseignement et de recherche français ou étrangers, des laboratoires publics ou privés. 
Elsevier Editorial System(tm) for International Journal of Radiation

Oncology*Biology*Physics Manuscript Draft

Manuscript Number: ROB-D-13-01170R2

Title: Combined Chemoradiotherapy with Twice Weekly Gemcitabine and Cisplatin for Organ Preservation in Muscle-invasive Bladder Cancer: Long-term Results of a Phase I Trial

Article Type: Full Length Article

Section/Category: Clinical Investigation - Genitourinary Cancers

Corresponding Author: Prof. David Azria, M.D., Ph.D.

Corresponding Author's Institution: CRLC Val d'Aurelle

First Author: David Azria, M.D., Ph.D.

Order of Authors: David Azria, M.D., Ph.D.; Olivier Riou; Xavier Rebillard; Simon Thezenas; Rodolphe Thuret; Pascal Fenoglietto; Damien Pouessel; Stephane Culine

Abstract: Purpose: Concomitant treatment with radiotherapy and cisplatin (CDDP) remains the gold standard for bladder preservation in case of muscle-invasive bladder cancer (MIBC). We present the long-term results of a phase I clinical trial to assess the association of gemcitabine (given twice/week) with CDDP and radiotherapy in this setting.

Methods and Materials: Patients with pT2-pT4 N0 M0 MIBC without hydronephrosis or diffuse carcinoma in situ were enrolled in this study. After maximal transurethral resection of the bladder tumor, patients received concomitantly radiotherapy (63 Gy in 1.8 fractions) and chemotherapy (20 $\mathrm{mg}$ CDDP $/ \mathrm{m}^{2} /$ day over 4 days every 21 days; and gemcitabine twice a week). The starting dose of gemcitabine was $15 \mathrm{mg} / \mathrm{m}^{2}$ with dose escalation to 20,25 and $30 \mathrm{mg} / \mathrm{m}^{2}$. The primary endpoint was the determination of the maximum tolerated dose (MTD). Secondary endpoints included the assessment of toxicity and tumor control.

Results: Fourteen patients were enrolled. Dose-limiting toxicity (DLT) occurred in two patients treated with $30 \mathrm{mg} / \mathrm{m}^{2}$ gemcitabine (grade 4 thrombocytopenia and severe impairment of the WHO performance status, respectively). Nine patients received the complete chemo-radiotherapy protocol. The recommended dose of gemcitabine was $25 \mathrm{mg} / \mathrm{m}^{2}$. The median follow-up was 53 months, and the overall and disease-specific survival rates were $62 \%$ and $77 \%$ at 5 years, respectively. Among the patients who received the complete treatment, bladder-intact survival was $76 \%$ at 5 years and the median overall survival 69.6 months.

Conclusions: This regimen was well-tolerated. The gemcitabine MTD was $25 \mathrm{mg} / \mathrm{m}^{2}$. Bladder preservation and disease control were promising. A multicenter phase II randomized trial is ongoing.

Suggested Reviewers:

Opposed Reviewers: 


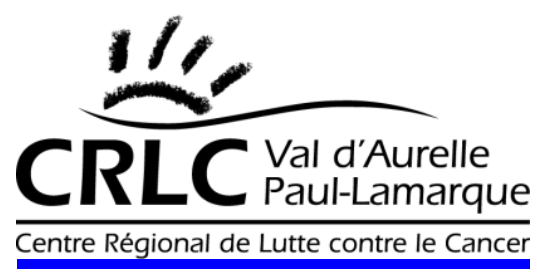

Centre Régional de Lutte contre le Cancer

\section{Inserm}

Instilut natlonal

de la sante et de la recherche modicale

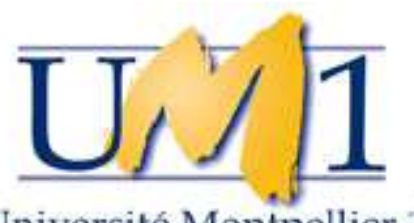

Université Montpellier 1

Montpellier November, $6^{\text {th }} 2013$

Editorial Office

International Journal of Radiation Oncology * Biology * Physics

8280 Willow Oaks Corporate Drive, Suite 500

Fairfax, VA 22031

USA

Dear Editor,

Here is the second revised version of our manuscript entitled "Combined Chemoradiotherapy with Twice Weekly Gemcitabine and Cisplatin for Organ Preservation in Muscle-invasive Bladder Cancer: Long-term Results of a Phase I Trial” (ROB-D-13-01170).

The manuscript has been review and corrected by the team of the Mount Sinai in New York.

We hope that all minor grammatical errors are now corrected.

Sincerely yours,

Pr David AZRIA 


\title{
Combined Chemoradiotherapy with Twice Weekly Gemcitabine and
}

\section{Cisplatin for Organ Preservation in Muscle-invasive Bladder Cancer:}

\section{Long-term Results of a Phase I Trial}

\author{
David Azria, MD, PhD, ${ }^{\ddagger,, \#, *}$ Olivier Riou, MD, ${ }^{\ddagger, \#}$ Xavier Rebillard, MD, ${ }^{\$}$ Simon \\ Thezenas, MSc, ${ }^{\Omega}$ Rodolphe Thuret, MD, PhD, ${ }^{\mathfrak{f}}$ Pascal Fenoglietto, MSc, ${ }^{\ddagger}$ Damien \\ Pouessel, MD, ${ }^{¥}$ and Stephane Culine, MD, PhD $^{\ddagger}$
}

\begin{abstract}
Department of Radiation Oncology and Radiophysics Unit, Montpellier Cancer Institute (ICM), Montpellier, France; ${ }^{\S}$ INSERM, U896, IRCM, Montpellier, France; ${ }^{\$}$ Department of Urology, Clinique Beausoleil, Montpellier, France;

${ }^{\Omega}$ Biostatistics Unit, Montpellier Cancer Institute, Montpellier, France; ${ }^{\ddagger}$ Department of Urology, Montpellier University Hospital, Montpellier, France; ${ }^{\sharp}$ Department of Medical Oncology, AP-HP Saint-Louis, Paris, France
\end{abstract}

${ }^{*}$ Reprint requests to: David Azria, MD, PhD, Department of Radiation Oncology, Montpellier Cancer Institute (ICM), 34298 Montpellier Cedex 5, France. Tel: + 334676131 32; Fax: + 334676131 35; E-mail : david.azria@icm.unicancer.fr

\# contributed equally to this work and should be considered as joint first authors

Presented in part at the American Society of Clinical Oncology Genito-Urinary (ASCO GU) Annual Meeting, San Francisco in 2010 and at the 53 ${ }^{\text {th }}$ ASTRO Annual Meeting, Boston in 2012.

Conflict of interest: none

Running title: Conservative bladder cancer treatment 


\section{Summary}

Concomitant radiotherapy and cisplatin (CCRT) remains the gold standard in the bladder preservation strategy in case of muscle invasive bladder cancer. We evaluated the role of adding gemcitabine given twice weekly to the standard CCRT. Among patients that received complete treatment, bladder-intact survival was $76 \%$ at 5 years and median overall survival was 69.6 months. The maximum-tolerated dose (MTD) of gemcitabine was $25 \mathrm{mg} / \mathrm{m}^{2}$. Bladder preservation and disease control were promising. 


\section{Combined Chemo-radiotherapy with Twice-weekly Gemcitabine and \\ Cisplatin for Organ Preservation in Muscle-invasive Bladder Cancer: \\ Long-term Results of a Phase I Trial}

Running title: Conservative bladder cancer treatment

\section{Summary}

Concomitant treatment with cisplatin and radiotherapy (CCRT) remains the gold standard for bladder preservation in the case of muscle-invasive bladder cancer. We evaluated the benefit of adding gemcitabine given twice a week to the standard CCRT. Among the patients who received the complete treatment, bladder-intact survival was $76 \%$ at 5 years and the median overall survival was 69.6 months. The gemcitabine maximum tolerated dose was $25 \mathrm{mg} / \mathrm{m}^{2}$. Bladder preservation and disease control were promising.

\footnotetext{
Abstract

Purpose: Concomitant treatment with radiotherapy and cisplatin (CDDP) remains the gold standard for bladder preservation in the case of muscle-invasive bladder cancer (MIBC). We present the long-term results of a phase I clinical trial to assess the association of twiceweekly gemcitabine with CDDP and radiotherapy in this setting.

Methods and Materials: Patients with pT2-pT4 N0 M0 MIBC without hydronephrosis or diffuse carcinoma in situ were enrolled in this study. After maximal transurethral resection of the bladder tumor, patients received concomitantly radiotherapy (63 Gy in 1.8 fractions) and chemotherapy (20 mg CDDP / $/ \mathrm{m}^{2} /$ day over 4 days every 21 days; and gemcitabine twice a week). The starting dose of gemcitabine was $15 \mathrm{mg} / \mathrm{m}^{2}$ with dose escalation to 20,25 and 30
} 
$\mathrm{mg} / \mathrm{m}^{2}$. The primary endpoint was the determination of the maximum tolerated dose (MTD). Secondary endpoints included the assessment of toxicity and tumor control.

Results: Fourteen patients were enrolled. Dose-limiting toxicity (DLT) occurred in two patients treated with $30 \mathrm{mg} / \mathrm{m}^{2}$ gemcitabine (grade 4 thrombocytopenia and severe impairment of the WHO performance status, respectively). Nine patients received the complete chemo-radiotherapy protocol. The recommended dose of gemcitabine was 25 $\mathrm{mg} / \mathrm{m}^{2}$. The median follow-up was 53 months, and the overall and disease-specific $\underline{5 \text {-year }}$ survival rates were $62 \%$ and $77 \%$ at 5 years, respectively. Among the patients who received the complete treatment, bladder-intact survival was $76 \%$ at 5 years and the median overall survival was 69.6 months.

Conclusions: This regimen was well-tolerated. The gemcitabine MTD was $25 \mathrm{mg} / \mathrm{m}^{2}$. Bladder preservation and disease control were promising. A multicenter phase II randomized trial is ongoing.

Keywords: bladder cancer; chemo-radiotherapy; gemcitabine; cisplatin; organ preservation 


\section{Introduction}

Concurrent chemo-radiotherapy (CCRT) for muscle-invasive transitional cell bladder cancer (MIBC) is an acceptable option for patients who are medically unfit for radical surgery as well as for patients initially selected for cystectomy. The standard CCRT includes a cisplatin (CDDP)-containing regimen and is associated with cystectomy-free survival rates between $42 \%$ and $55 \%$ at 5 years, depending on the initial tumor stage (1-6).

Gemcitabine has been shown to be active ineffective in treating bladder cancer in combination with CDDP in neoadjuvant and metastatic settings $(7,8)$. In addition, gemcitabine has significant radiosensitizing activity in various cancer cell lines $(9,10)$, including those derived from bladder tumors (11). The effectiveness of gemcitabine-based CCRT has been widely reported for many different epithelial tumors $(12,13)$. Gemcitabine sensitizing activity occurs at sub-cytotoxic doses and $_{2}$ the mechanism involves depletion of the deoxyribonucleoside triphosphate pools, particularly deoxyadenosine triphosphate (dATP) (14). Consecutive studies confirmed that weekly doses ranging from 150 to $450 \mathrm{mg} / \mathrm{m}^{2}$ can be successfully used in combination with radiotherapy (10). Furthermore, as preclinical studies showed that even lower doses of gemcitabine effectively radiosensitize cells for up to 72 hours $(15,16)$, several trials were carried out to test lower gemcitabine doses in clinical settings. Specifically, gemcitabine (twice a week) in combination with radiotherapy (and no CDDP) was well tolerated by patients with MIBC (17). The maximum tolerated dose (MTD) was $27 \mathrm{mg} / \mathrm{m}^{2}$ twice a week with 60 Gy delivered to the bladder over 6 weeks.

As the optimal CCRT should contain $\mathrm{CDDP}_{2}$ and gemcitabine potentially radiosensitizes bladder cancer cells, we decided to conduct a phase I trial in which patients with initially operable MIBC were treated with gemcitabine twice/week concomitantly with CDDP and radiotherapy. 


\section{Methods and Materials}

Our local institutional review board approved the protocol, and written informed consent was obtained from all patients. This study was registered at ClinicalTrials.gov, number NCT00556621.

\section{Study design and endpoints}

This was a Phase I dose-finding study to determine the MTD and to record acute and late toxicity following CCRT with gemcitabine and CDDP in patients with operable MIBC. The MTD was defined as the gemcitabine dose associated with dose-limiting toxicity (DLT) occurring in 3 out of 6 patients or in 2 out of 3 patients (in this case, an accrual of 3 more patients to the previous dose level was planned).

DLT was defined as: grade 2 or higher pulmonary toxicity, according to the third version of the Common Toxicity Criteria for Adverse Events scale (CTCAE 3.0); all other nonhematological grade 3 or higher CTCAE 3.0 adverse events (except nausea and vomiting); grade 4 thrombocytopenia, grade 3 thrombocytopenia lasting more than seven days or complicated by hemorrhage; grade 4 neutropenia for more than seven days, febrile neutropenia or severe infection.

\section{Patients ${ }^{9}$ selection}

Only patients with histologically confirmed MIBC after macroscopically complete transurethral resection of the bladder tumor (TURBT) were enrolled. A second TURBT was carried out if residual microscopic tumor cells were detected around the primary tumor site. 
At diagnosis, a CT scan of the chest, abdomen and pelvis and a bone scan were performed to rule out metastatic disease and to stage the tumor in all patients. The pretreatment evaluation included a physical examination and routine laboratory tests, including complete blood cell count, electrolyte, blood urea nitrogen, creatinine and glucose levels and liver function.

Inclusion criteria were: pT2-pT4a MIBC with microscopically complete resection after the first or second TURBT; no macroscopically visible lesions in the pelvic nodes (N0) or distant metastases (M0); absence of carcinoma in situ (CIS); no hydronephrosis; (v) Karnofsky performance score $\geq 70 \%$; life expectancy $\geq 6$ months; adequate bone marrow reserve (defined as: pretreatment absolute neutrophil count $\geq 1500 / \mu \mathrm{L}$, hemoglobin level $\geq 10 \mathrm{~g} / \mathrm{dL}$, and platelet count $\geq 100,000 / \mu \mathrm{L}) ;$ creatinine clearance $\geq 60 \mathrm{~mL} / \mathrm{min} ;$ bilirubin and AST $\leq 3$ and 4 times the institutional upper limits of normal, respectively); age $\geq 18$ years; signed informed consent form.

Pregnant or breast-feeding patients were excluded as well as patients with previous radiotherapy or chemotherapy treatments (except anterior intra-bladder treatment for localized CIS), or history of malignancies other than adequately treated basal cell or squamous cell skin cancer or in situ cervical carcinoma.

\section{Treatment plan}

The treatment was started within eight weeks after complete TURBT.

\section{Radiotherapy}

Patients underwent CT-based virtual simulation using $2.5 \mathrm{~mm}$ thick slices obtained at $2.5 \mathrm{~mm}$ intervals. Patients were treated in a supine position with empty bladder.

During the first CCRT part a fractionated dose of $45 \mathrm{~Gy}(1.8 \mathrm{~Gy} /$ fraction/day in 25 fractions, over five weeks) was administered to the small pelvis (from S1-S2 to the obturator foramen or 
the ischiatic tuberosity in case of bladder neck or prostatic urethra invasion) using a 4-field box technique. The clinical target volume included the bladder, the obturators and the internal and external iliac lymph nodes. During the second CCRT part, 18 Gy (1.8 Gy/fraction/day in 10 fractions, over two weeks) were delivered to a clinical target volume limited to the initial tumor volume, using reduced-box or opposed lateral or antero-posterior fields. A $1.5-\mathrm{cm}$ margin was added to the clinical target volume to allow for geometric uncertainties (planning target volume).

Patients were treated with $18 \mathrm{MV}$ photon beams. Dose prescription was in accordance with the ICRU 62 report for each patient.

\section{Cisplatin (CDDP)}

CDDP was given at a dose of $20 \mathrm{mg} / \mathrm{m}^{2} /$ day by continuous intravenous.. . infusion during 4 consecutive days (from day 2 to day 5 and from day 23 to day 26 during the first CCRT part and from day 2 to 5 of the second CCRT part). Patients were hydrated and received antiemetic drugs according to our in-house protocol.

\section{Gemcitabine}

Gemcitabine was administered intravenously in $50-100 \mathrm{~mL}$ of normal saline over $30 \mathrm{~min} 2$ to 6 hours prior to irradiation and two times a week, on day 2, 5, 9, 12, 16, 19, 23, 26, 30 and 33 (first CCRT part) and on day 2, 5, 9 and 12 (second CCRT part).

The starting gemcitabine dose was $15 \mathrm{mg} / \mathrm{m}^{2}$ twice/week with planned dose escalation of 5 $\mathrm{mg} / \mathrm{m}^{2}$ every two weeks unless the MTD was reached. Dose escalation was not allowed in the same patient. 


\section{Dose modification}

Dose adjustments were based on the weekly absolute neutrophil count (ANC) and platelet count, assessed no later than 36 hours before the gemcitabine infusion and on the clinical assessment of non-hematological toxicities. Gemcitabine dose was not modified if the ANC was $>1000 / \mu \mathrm{L}$ and/or the platelet count was $>100,000 / \mu \mathrm{L}$. In the case of an ANC between 500 and $1000 / \mu \mathrm{L}$ and a platelet count between 50,000 and $100,000 / \mu \mathrm{L}$, gemcitabine was not administered. In the case of an even lower ANC or platelet count, chemotherapy was definitively stopped.

Reduction of the creatinine clearance level between 40 and $60 \mathrm{~mL} / \mathrm{min}$ entailed a $50 \%$ decrease of the CDDP dose. In the case of even lower values, CDDP was stopped for the entire course.

If a DLT occurred, chemotherapy was stopped, but radiotherapy could be continued based on the investigator's assessment.

\section{Follow-up, toxicity and response evaluation}

During treatment, patients were seen weekly at the Radiation Oncology Department and weight, toxicity and complete blood count (including blood cell differential and platelet count twice a week before each gemcitabine injection) were recorded.

An evaluation by cystoscopy and TURBT under general anesthesia was performed during the third week after the end of the first CCRT part. Complete response (CR) was defined as the absence of any macroscopic or microscopic lesion confirmed by the pathologist. In the case of residual disease, total cystectomy was proposed to the patient. In the case of CR, the second CCRT part was started as soon as possible and no later than four weeks from the completion of the first cycle. The final evaluation was done by cystoscopy and TURBT six to eight weeks after CCRT completion. 
After the end of the treatment, patients were clinically assessed every month for the first four months and then once every six months (clinical examination, CT scan with contrast and cystoscopy).

\section{Statistical analysis}

Data were analyzed using the STATA software version 8.0 (Stata Corporation, College Station, TX, USA). Survival probabilities were estimated using the actuarial or the KaplanMeier method and the following definitions.

Overall survival (OS): the event was death from any cause. The time to OS was the interval between treatment initiation and death, or the most recent follow-up if no event occurred. Disease-specific survival (DSS): the event was death attributable to bladder cancer. The time to DSS was the interval between treatment initiation and death from bladder cancer, or the most recent follow-up if no event occurred.

Bladder-intact survival (BIS): the event was cystectomy for any reason or death from any cause. The time to BIS was the interval between treatment initiation and cystectomy or death (whichever was shorter), or the most recent follow-up if no event occurred. 


\section{Results}

\section{Patients' characteristics and response to treatment}

Fourteen patients with a median age of 72 years (range 51-83) were included in this study between June 2005 and June 2009. The patients' characteristics are listed in Table 1.

One patient was excluded from the protocol on day 5 of the first CCRT cycle due to poor compliance (intravenous drip pulled out and aggressive behavior). He was then treated offprotocol with radiotherapy (total dose: $63 \mathrm{~Gy}$; complete response after the first cycle) and CDDP alone (modified dose of $40 \mathrm{mg} / \mathrm{m}^{2} /$ day on day 23 and 24 of the first cycle and on day 2 and 3 of the second cycle). He was included in the follow-up assessment ("intent-to-treat" approach).

All patients received the first radiotherapy part (45 Gy) and 12 patients (86\%) completed the whole radiotherapy protocol (45 Gy $+18 \mathrm{~Gy})$. Nine patients $(64 \%)$ completed the whole CCRT course with gemcitabine and CDDP.

At the TURBT evaluation after the first CCRT part, ten patients showed CR $(71 \%$ of the included patients). One patient had stable disease and underwent cystectomy. Two patients (14\%) had progressive disease: one underwent cystectomy and the other (who refused radical surgery) continued with the second CCRT part with CR at the end of the treatment. One patient (7\%) could not be evaluated (excluded from the protocol).

The intact bladder preservation rate was $86 \%$ for all included patients (12 out of 14 patients).

\section{Toxicity}

Table 2 reports the acute adverse events that occurred outside the radiation field and could thus be considered as related to chemotherapy. They were mainly hematological disorders, 
digestive alterations and asthenia. Only two of these adverse events were considered as-DLT (grade 4 thrombocytopenia and grade 3 asthenia). Table 3 reports the adverse events due to radiotherapy in the 14 patients. As expected, urinary discomfort and diarrhea were the two main acute adverse events. No in-field severe events (to be considered as DLT) were observed. Table 4 details the gemcitabine dose, the time of appearance of toxicity, the toxicity grade and DLT leading to chemotherapy delay. DLT was confirmed in two patients. One was a grade 4 thrombocytopenia at day 16 after four injections of $30 \mathrm{mg} / \mathrm{m}^{2}$ gemcitabine and one 4-day continuous infusion of CDDP. Chemotherapy was stopped, and the patient spontaneously recovered. CR was achieved after radiotherapy alone. The second DLT occurred at day 26 and consisted of an overall alteration of the performance status, arrhythmia (auricular fibrillation) and global edema. The patient had already received eight injections of $30 \mathrm{mg} / \mathrm{m}^{2}$ gemcitabine and two 4-day continuous infusions of CDDP. Only radiotherapy was continued and CR was achieved.

\section{Follow-up and survival}

The median follow-up was 53 months (4.4 years) for the whole cohort. Two patients died of progressive metastatic disease. One died of metastatic disease after salvage cystectomy for progressive local disease during the first CCRT part. The second one died of metastatic disease in the first year although the tumor was locally controlled.

Four patients died of undercurrent diseases. One patient died of an ischemic stroke after treatment completion, another of metastatic breast disease and the other two from cardiovascular disease.

The median OS for all patients was 5.8 years. The actuarial OS rates were $79 \%$ (at 2 years), $71 \%$ (3 years) and 62\% (5 years). The median DSS was not reached, but the actuarial DSS 
rate was $77 \%$ at five years. The median BIS was 5.8 years for all patients. The actuarial BIS rates were $64 \%$ at three years and $56 \%$ at five years (Fig.1).

The nine patients who received the whole CCRT protocol had a median OS of 5.8 years, a 5year actuarial DSS rate of $89 \%$ and SIB of $89 \%$ (at three years) and $76 \%$ (five years).

No salvage cystectomy for local tumor recurrence or toxicity was performed during the follow-up period and no significant long-term late toxicity occurred during the follow-up. 


\section{Discussion}

CCRT with CDDP is the most studied protocol for the conservative management of MIBC. Although other systemic drugs (such as 5-FU, taxanes, or gemcitabine) have been already added to CDDP to increase local control, the combination of fractionated pelvic radiotherapy,

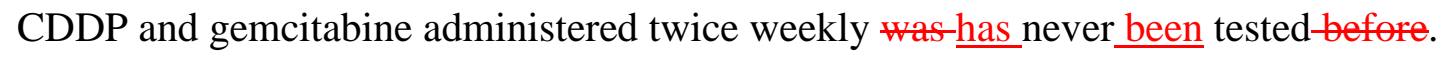

We present here the long-term results of our phase I study to assess gemcitabine as a potent radiosensitizer in combination with CDDP for the conservative management of MIBC. A standard 3+3 Phase I dose escalation schedule was planned as no data was available on the twice-weekly gemcitabine and CDDP combination as radiosensitizers. A long-term follow-up of our patients was required as gemcitabine may potentially increase late toxicity, when combined with pelvic radiotherapy. However, the observed toxicity was acceptable with just one grade four thrombocytopenia and one grade four asthenia considered as DLT. No in-field severe event occurred. Regarding the efficacy, our long-term results are quite similar to previously published data in this setting $(18,19)$. Indeed, among patients that received the complete treatment, BIS was $76 \%$ at five years and the median OS was 69.6 months.

Another phase I trial combining gemcitabine and RT with long-term results has been already published, but the pelvic fields were not treated thus making difficult any comparison (20). As usually performed, especially in the trials of the Radiation Therapy Oncology Group (21), we included the pelvic lymph nodes in the radiation field because lymph node involvement is about $20 \%$ and $40 \%$ in pT2 and pT3 tumors, respectively (22). Another phase I study including pelvic fields with gemcitabine reported high toxicity rates and had to be amended to a de-escalation study, with a $25 \mathrm{mg} / \mathrm{m}^{2}$ decrease at each level (23). Consequently, the toxicity observed in this phase I trial should absolutely be interpreted by taking into account this large radiation volume that increases the risk of interactions between ionizing radiation, gemcitabine and CDDP. 
Kent et al. conducted a phase I study on gemcitabine given twice weekly without CDDP and with 60 Gy of radiation after TURBT (17). DLT included deep venous thrombosis, diarrhea, abnormal liver function tests and edema. The recommended dose in this trial was $27 \mathrm{mg} / \mathrm{m}^{2}$ twice-weekly, which is close to the MTD found in our study ( $25 \mathrm{mg} / \mathrm{m}^{2}$ given twice-weekly). Besides, Caffo et al. performed a phase I study on conservative radiotherapy with weekly gemcitabine and $100 \mathrm{mg} / \mathrm{m}^{2}$ CDDP every three weeks (18). Starting with $200 \mathrm{mg} / \mathrm{m}^{2} /$ week gemcitabine, they escalated the dose up to $500 \mathrm{mg} / \mathrm{m}^{2} /$ week, with two DLT: one death and one intestinal perforation. The gemcitabine recommended dose of $400 \mathrm{mg} / \mathrm{m}^{2} /$ week was then evaluated in a phase II study that was unfortunately prematurely closed due to low accrual. The radiotherapy dose was $54 \mathrm{~Gy}(1.8 \mathrm{~Gy} /$ fraction $)$ in both trials. A pooled analysis of the two trials on 26 patients was published in 2010 with a median follow-up of 74 months (24). The 5year clinical outcomes were a $70.1 \%$ OS rate, a $78.9 \%$ DSS rate and a $73.8 \%$ BIS rate. Although this association was effective as all evaluable patients were disease-free at the time of the cystoscopic evaluation, concerns regarding toxicity with the weekly gemcitabine schedule remain. Indeed, intestinal toxicities in the phase I study and neutropenia in the phase II study frequently occurred (even after omission of the gemcitabine injection at day 15 and 36) and for that reason we chose a twice-weekly schedule allowing for lower doses of gemcitabine.

In our study, we used standard radiotherapy fractionation (1.8 Gy/fraction/day 5 days a week). However, another group studied gemcitabine radiosensitization with hypofractionated chemoradiotherapy (52.5 Gy in 20 fractions over 28 days) in a phase I study and found a MTD of $100 \mathrm{mg} / \mathrm{m}^{2}$ when delivered only once a week and without CDDP (25). The consecutive phase II trial included 50 patients with transitional cell carcinoma staged T2-3 N0 M0 (19). All patients completed the radiotherapy course; 46 (92\%) tolerated all four gemcitabine cycles. Two patients stopped after two cycles, and two after three cycles, due to bowel toxicity. Forty-four (88\%) achieved a complete endoscopic response. At a median follow-up of 36 
months (range, 15- 62 months), 36 patients were alive and 32 had a functional and intact bladder. By using Kaplan-Meier analyses, the 3-year cancer-specific survival was $82 \%$, and OS was $75 \%$. Nevertheless, these results were obtained with hypofractionated conformal radiotherapy that used a four-field plan with multi-leaf collimators, delivered to the whole bladder and a minimum $1.5-\mathrm{cm}$ margin. Finally, these regimens are clearly manageable for patients with comorbidities but we are still convinced that CDDP presents a strong addedvalue in fit patients.

The results presented here allowed us to determine the gemcitabine MTD when given twice a week in combination with CDDP and radiotherapy. Although bladder preservation and disease control results are promising, this remains a phase I study with only 14 patients and a further validation in a larger cohort is needed. In view of these findings, a French phase II randomized multi-institutional trial named GETUG V04 started in 2011 to assess the efficacy and tolerability of this new combination strategy with gemcitabine twice-weekly compared to the standard CCRT schedule with CDDP alone (NCT01495676). 


\section{References}

1. Dunst J, Sauer R, Schrott KM, et al. Organ-sparing treatment of advanced bladder cancer: a 10-year experience. Int J Radiat Oncol Biol Phys 1994;30:261-266.

2. Tester W, Porter A, Asbell S, et al. Combined modality program with possible organ preservation for invasive bladder carcinoma: results of RTOG protocol 85-12. Int J Radiat Oncol Biol Phys 1993;25:783-790.

3. Kaufman DS, Winter KA, Shipley WU, et al. The initial results in muscle-invading bladder cancer of RTOG 95-06: phase I/II trial of transurethral surgery plus radiation therapy with concurrent cisplatin and 5-fluorouracil followed by selective bladder preservation or cystectomy depending on the initial response. Oncologist 2000;5:471-476.

4. Housset M, Maulard C, Chretien Y, et al. Combined radiation and chemotherapy for invasive transitional-cell carcinoma of the bladder: a prospective study. J Clin Oncol $1993 ; 11: 2150-2157$.

5. Rödel C, Grabenbauer GG, Kühn R, et al. Combined-modality treatment and selective organ preservation in invasive bladder cancer: long-term results. J Clin Oncol 2002;20:3061-3071.

6. Ott OJ, Rödel C, Weiss C, et al. Radiochemotherapy for bladder cancer. Clin Oncol 2009;21:557-565.

7. von der Maase H, Sengelov L, Roberts JT, et al. Long-term survival results of a randomized trial comparing gemcitabine plus cisplatin, with methotrexate, vinblastine, doxorubicin, plus cisplatin in patients with bladder cancer. J Clin Oncol 2005;23:46024608.

8. Kaufman DS, Shipley WU, Feldman AS. Bladder cancer. Lancet 2009;374:239-249.

9. Shewach DS, Lawrence TS. Radiosensitization of human solid tumor cell lines with gemcitabine. Semin Oncol 1996;23:65-71. 
10. Azria $\mathrm{D}$, Jacot $\mathrm{W}$, Prost $\mathrm{P}$, et al. Gemcitabine and ionizing radiations: radiosensitization or radio-chemotherapy combination. Bull Cancer 2002;89:369-379.

11. Sangar VK, Cowan R, Margison GP, et al. An evaluation of gemcitabines differential radiosensitising effect in related bladder cancer cell lines. Br J Cancer 2004;90:542-548.

12. Huguet F, Girard N, Guerche CS-E, et al. Chemoradiotherapy in the management of locally advanced pancreatic carcinoma: a qualitative systematic review. J Clin Oncol 2009;27:2269-2277.

13. Girard N, Mornex F, Douillard J-Y, et al. Is neoadjuvant chemoradiotherapy a feasible strategy for stage IIIA-N2 non-small cell lung cancer? Mature results of the randomized IFCT-0101 phase II trial. Lung Cancer 2010;69:86-93.

14. Shewach DS, Hahn TM, Chang E, et al. Metabolism of 2',2'-difluoro-2'-deoxycytidine and radiation sensitization of human colon carcinoma cells. Cancer Res 1994;54:32183223

15. Lawrence TS, Chang EY, Hahn TM, et al. Radiosensitization of pancreatic cancer cells by 2',2'-difluoro-2'-deoxycytidine. Int J Radiat Oncol Biol Phys 1996;34:867-872.

16. Lawrence TS, Chang EY, Hahn TM, et al. Delayed radiosensitization of human colon carcinoma cells after a brief exposure to 2',2'-difluoro-2'-deoxycytidine (Gemcitabine). Clin Cancer Res 1997;3:777-782.

17. Kent E, Sandler H, Montie J, et al. Combined-Modality Therapy With Gemcitabine and Radiotherapy As a Bladder Preservation Strategy: Results of a Phase I Trial. J Clin Oncol $2004 ; 22: 2540-2545$.

18. Caffo O, Fellin G, Graffer U, et al. Phase I study of gemcitabine and radiotherapy plus cisplatin after transurethral resection as conservative treatment for infiltrating bladder cancer. Int J Radiat Oncol Biol Phys 2003;57:1310-6. 
19. Choudhury A, Swindell R, Logue JP, et al. Phase II Study of Conformal Hypofractionated Radiotherapy With Concurrent Gemcitabine in Muscle-Invasive Bladder Cancer. Journal of Clinical Oncology. 2011;4;29(6):733-8.

20. Oh KS, Soto DE, Smith DC, et al. Combined-Modality Therapy With Gemcitabine and Radiation Therapy as a Bladder Preservation Strategy: Long-Term Results of a Phase I Trial. Int J Radiat Oncol Biol Phys 2009;74:511-517.

21. Mitin T, Hunt D, Shipley WU, et al. Transurethral surgery and twice-daily radiation plus paclitaxel-cisplatin or fluorouracil-cisplatin with selective bladder preservation and adjuvant chemotherapy for patients with muscle invasive bladder cancer (RTOG 0233): a randomised multicentre phase 2 trial. Lancet Oncol 2013;14:863-872.

22. Tarin TV, Power NE, Ehdaie B, et al. Lymph node-positive bladder cancer treated with radical cystectomy and lymphadenectomy: effect of the level of node positivity. Eur Urol 2012;61:1025-1030.

23. Borut K, Lijana ZK. Phase I study of radiochemotherapy with gemcitabine in invasive bladder cancer. Radiother Oncol 2012;102:412-415.

24. Caffo O, Fellin G, Graffer U, et al. Gemcitabine and radiotherapy plus cisplatin after transurethral resection as conservative treatment for infiltrating bladder cancer. Cancer 2011;117:1190-1196.

25. Sangar V, Mcbain C, Lyons J, et al. Phase I study of conformal radiotherapy with concurrent gemcitabine in locally advanced bladder cancer. Int J Radiat Oncol Biol Phys $2005 ; 61: 420-425$. 


\section{Figure legends}

Fig. 1 Survival curves obtained using the Kaplan-Meier method for the overall survival (OS), disease-specific survival (DSS) and bladder-intact survival (BIS). 


\title{
Combined Chemo-radiotherapy with Twice-weekly Gemcitabine and Cisplatin for Organ Preservation in Muscle-invasive Bladder Cancer: Long-term Results of a Phase I Trial
}

Running title: Conservative bladder cancer treatment

\section{Summary}

Concomitant treatment with cisplatin and radiotherapy (CCRT) remains the gold standard for bladder preservation in the case of muscle-invasive bladder cancer. We evaluated the benefit of adding gemcitabine given twice a week to the standard CCRT. Among the patients who received the complete treatment, bladder-intact survival was $76 \%$ at 5 years and the median overall survival was 69.6 months. The gemcitabine maximum tolerated dose was $25 \mathrm{mg} / \mathrm{m}^{2}$. Bladder preservation and disease control were promising.

\begin{abstract}
Purpose: Concomitant treatment with radiotherapy and cisplatin (CDDP) remains the gold standard for bladder preservation in the case of muscle-invasive bladder cancer (MIBC). We present the long-term results of a phase I clinical trial to assess the association of twiceweekly gemcitabine with CDDP and radiotherapy in this setting.

Methods and Materials: Patients with pT2-pT4 N0 M0 MIBC without hydronephrosis or diffuse carcinoma in situ were enrolled in this study. After maximal transurethral resection of the bladder tumor, patients received concomitantly radiotherapy (63 Gy in 1.8 fractions) and chemotherapy (20 mg CDDP $/ \mathrm{m}^{2} /$ day over 4 days every 21 days; and gemcitabine twice a week). The starting dose of gemcitabine was $15 \mathrm{mg} / \mathrm{m}^{2}$ with dose escalation to 20,25 and 30
\end{abstract}


$\mathrm{mg} / \mathrm{m}^{2}$. The primary endpoint was the maximum tolerated dose (MTD). Secondary endpoints included toxicity and tumor control.

Results: Fourteen patients were enrolled. Dose-limiting toxicity (DLT) occurred in two patients treated with $30 \mathrm{mg} / \mathrm{m}^{2}$ gemcitabine (grade 4 thrombocytopenia and severe impairment of the WHO performance status, respectively). Nine patients received the complete chemo-radiotherapy protocol. The recommended dose of gemcitabine was 25 $\mathrm{mg} / \mathrm{m}^{2}$. The median follow-up was 53 months, and the overall and disease-specific 5-year survival rates were $62 \%$ and $77 \%$, respectively. Among the patients who received the complete treatment, bladder-intact survival was $76 \%$ at 5 years and the median overall survival was 69.6 months.

Conclusions: This regimen was well-tolerated. The gemcitabine MTD was $25 \mathrm{mg} / \mathrm{m}^{2}$. Bladder preservation and disease control were promising. A multicenter phase II randomized trial is ongoing.

Keywords: bladder cancer; chemo-radiotherapy; gemcitabine; cisplatin; organ preservation 


\section{Introduction}

Concurrent chemo-radiotherapy (CCRT) for muscle-invasive transitional cell bladder cancer (MIBC) is an acceptable option for patients who are medically unfit for radical surgery as well as for patients initially selected for cystectomy. The standard CCRT includes a cisplatin (CDDP)-containing regimen and is associated with cystectomy-free survival rates between $42 \%$ and $55 \%$ at 5 years, depending on the initial tumor stage (1-6).

Gemcitabine has been shown to be effective in treating bladder cancer in combination with CDDP in neoadjuvant and metastatic settings $(7,8)$. In addition, gemcitabine has significant radiosensitizing activity in various cancer cell lines $(9,10)$, including those derived from bladder tumors (11). The effectiveness of gemcitabine-based CCRT has been widely reported for many different epithelial tumors $(12,13)$. Gemcitabine sensitizing activity occurs at subcytotoxic doses, and the mechanism involves depletion of the deoxyribonucleoside triphosphate pools, particularly deoxyadenosine triphosphate (dATP) (14). Consecutive studies confirmed that weekly doses ranging from 150 to $450 \mathrm{mg} / \mathrm{m}^{2}$ can be successfully used in combination with radiotherapy (10). Furthermore, as preclinical studies showed that even lower doses of gemcitabine effectively radiosensitize cells for up to 72 hours $(15,16)$, several trials were carried out to test lower gemcitabine doses in clinical settings. Specifically, gemcitabine (twice a week) in combination with radiotherapy (and no CDDP) was well tolerated by patients with MIBC (17). The maximum tolerated dose (MTD) was $27 \mathrm{mg} / \mathrm{m}^{2}$ twice a week with 60 Gy delivered to the bladder over 6 weeks.

As the optimal CCRT should contain CDDP, and gemcitabine potentially radiosensitizes bladder cancer cells, we decided to conduct a phase I trial in which patients with initially operable MIBC were treated with gemcitabine twice/week concomitantly with CDDP and radiotherapy. 


\section{Methods and Materials}

Our local institutional review board approved the protocol, and written informed consent was obtained from all patients. This study was registered at ClinicalTrials.gov, number NCT00556621.

\section{Study design and endpoints}

This was a Phase I dose-finding study to determine the MTD and to record acute and late toxicity following CCRT with gemcitabine and CDDP in patients with operable MIBC. The MTD was defined as the gemcitabine dose associated with dose-limiting toxicity (DLT) occurring in 3 out of 6 patients or in 2 out of 3 patients (in this case, an accrual of 3 more patients to the previous dose level was planned).

DLT was defined as: grade 2 or higher pulmonary toxicity, according to the third version of the Common Toxicity Criteria for Adverse Events scale (CTCAE 3.0); all other nonhematological grade 3 or higher CTCAE 3.0 adverse events (except nausea and vomiting); grade 4 thrombocytopenia, grade 3 thrombocytopenia lasting more than seven days or complicated by hemorrhage; grade 4 neutropenia for more than seven days, febrile neutropenia or severe infection.

\section{Patient selection}

Only patients with histologically confirmed MIBC after macroscopically complete transurethral resection of the bladder tumor (TURBT) were enrolled. A second TURBT was carried out if residual microscopic tumor cells were detected around the primary tumor site. 
At diagnosis, a CT scan of the chest, abdomen and pelvis and a bone scan were performed to rule out metastatic disease and to stage the tumor in all patients. The pretreatment evaluation included a physical examination and routine laboratory tests, including complete blood cell count, electrolyte, blood urea nitrogen, creatinine and glucose levels and liver function.

Inclusion criteria were: pT2-pT4a MIBC with microscopically complete resection after the first or second TURBT; no macroscopically visible lesions in the pelvic nodes (N0) or distant metastases (M0); absence of carcinoma in situ (CIS); no hydronephrosis; (v) Karnofsky performance score $\geq 70 \%$; life expectancy $\geq 6$ months; adequate bone marrow reserve (defined as: pretreatment absolute neutrophil count $\geq 1500 / \mu \mathrm{L}$, hemoglobin level $\geq 10 \mathrm{~g} / \mathrm{dL}$, and platelet count $\geq 100,000 / \mu \mathrm{L}) ;$ creatinine clearance $\geq 60 \mathrm{~mL} / \mathrm{min} ;$ bilirubin and AST $\leq 3$ and 4 times the institutional upper limits of normal, respectively); age $\geq 18$ years; signed informed consent form.

Pregnant or breast-feeding patients were excluded as well as patients with previous radiotherapy or chemotherapy treatments (except anterior intra-bladder treatment for localized CIS), or history of malignancies other than adequately treated basal cell or squamous cell skin cancer or in situ cervical carcinoma.

\section{Treatment plan}

The treatment was started within eight weeks after complete TURBT.

\section{Radiotherapy}

Patients underwent CT-based virtual simulation using $2.5 \mathrm{~mm}$ thick slices obtained at $2.5 \mathrm{~mm}$ intervals. Patients were treated in a supine position with empty bladder.

During the first CCRT part a fractionated dose of $45 \mathrm{~Gy}(1.8 \mathrm{~Gy} /$ fraction/day in 25 fractions, over five weeks) was administered to the small pelvis (from S1-S2 to the obturator foramen or 
the ischiatic tuberosity in case of bladder neck or prostatic urethra invasion) using a 4-field box technique. The clinical target volume included the bladder, the obturators and the internal and external iliac lymph nodes. During the second CCRT part, 18 Gy (1.8 Gy/fraction/day in 10 fractions, over two weeks) were delivered to a clinical target volume limited to the initial tumor volume, using reduced-box or opposed lateral or antero-posterior fields. A $1.5-\mathrm{cm}$ margin was added to the clinical target volume to allow for geometric uncertainties (planning target volume).

Patients were treated with $18 \mathrm{MV}$ photon beams. Dose prescription was in accordance with the ICRU 62 report for each patient.

\section{Cisplatin (CDDP)}

CDDP was given at a dose of $20 \mathrm{mg} / \mathrm{m}^{2} /$ day by continuous intravenous infusion during 4 consecutive days (from day 2 to day 5 and from day 23 to day 26 during the first CCRT part and from day 2 to 5 of the second CCRT part). Patients were hydrated and received antiemetic drugs according to our in-house protocol.

\section{Gemcitabine}

Gemcitabine was administered intravenously in $50-100 \mathrm{~mL}$ of normal saline over $30 \mathrm{~min} 2$ to 6 hours prior to irradiation and two times a week, on day 2, 5, 9, 12, 16, 19, 23, 26, 30 and 33 (first CCRT part) and on day 2, 5, 9 and 12 (second CCRT part).

The starting gemcitabine dose was $15 \mathrm{mg} / \mathrm{m}^{2}$ twice/week with planned dose escalation of 5 $\mathrm{mg} / \mathrm{m}^{2}$ every two weeks unless the MTD was reached. Dose escalation was not allowed in the same patient. 


\section{Dose modification}

Dose adjustments were based on the weekly absolute neutrophil count (ANC) and platelet count, assessed no later than 36 hours before the gemcitabine infusion and on the clinical assessment of non-hematological toxicities. Gemcitabine dose was not modified if the ANC was $>1000 / \mu \mathrm{L}$ and/or the platelet count was $>100,000 / \mu \mathrm{L}$. In the case of an ANC between 500 and $1000 / \mu \mathrm{L}$ and a platelet count between 50,000 and $100,000 / \mu \mathrm{L}$, gemcitabine was not administered. In the case of an even lower ANC or platelet count, chemotherapy was definitively stopped.

Reduction of the creatinine clearance level between 40 and $60 \mathrm{~mL} / \mathrm{min}$ entailed a $50 \%$ decrease of the CDDP dose. In the case of even lower values, CDDP was stopped for the entire course.

If a DLT occurred, chemotherapy was stopped, but radiotherapy could be continued based on the investigator's assessment.

\section{Follow-up, toxicity and response evaluation}

During treatment, patients were seen weekly at the Radiation Oncology Department and weight, toxicity and complete blood count (including blood cell differential and platelet count twice a week before each gemcitabine injection) were recorded.

An evaluation by cystoscopy and TURBT under general anesthesia was performed during the third week after the end of the first CCRT part. Complete response (CR) was defined as the absence of any macroscopic or microscopic lesion confirmed by the pathologist. In the case of residual disease, total cystectomy was proposed to the patient. In the case of CR, the second CCRT part was started as soon as possible and no later than four weeks from the completion of the first cycle. The final evaluation was done by cystoscopy and TURBT six to eight weeks after CCRT completion. 
After the end of the treatment, patients were clinically assessed every month for the first four months and then once every six months (clinical examination, CT scan with contrast and cystoscopy).

\section{Statistical analysis}

Data were analyzed using the STATA software version 8.0 (Stata Corporation, College Station, TX, USA). Survival probabilities were estimated using the actuarial or the KaplanMeier method and the following definitions.

Overall survival (OS): the event was death from any cause. The time to OS was the interval between treatment initiation and death, or the most recent follow-up if no event occurred. Disease-specific survival (DSS): the event was death attributable to bladder cancer. The time to DSS was the interval between treatment initiation and death from bladder cancer, or the most recent follow-up if no event occurred.

Bladder-intact survival (BIS): the event was cystectomy for any reason or death from any cause. The time to BIS was the interval between treatment initiation and cystectomy or death (whichever was shorter), or the most recent follow-up if no event occurred. 


\section{Results}

\section{Patients' characteristics and response to treatment}

Fourteen patients with a median age of 72 years (range 51-83) were included in this study between June 2005 and June 2009. The patients' characteristics are listed in Table 1.

One patient was excluded from the protocol on day 5 of the first CCRT cycle due to poor compliance (intravenous drip pulled out and aggressive behavior). He was then treated offprotocol with radiotherapy (total dose: $63 \mathrm{~Gy}$; complete response after the first cycle) and CDDP alone (modified dose of $40 \mathrm{mg} / \mathrm{m}^{2} /$ day on day 23 and 24 of the first cycle and on day 2 and 3 of the second cycle). He was included in the follow-up assessment ("intent-to-treat" approach).

All patients received the first radiotherapy part (45 Gy) and 12 patients (86\%) completed the whole radiotherapy protocol (45 Gy $+18 \mathrm{~Gy})$. Nine patients $(64 \%)$ completed the whole CCRT course with gemcitabine and CDDP.

At the TURBT evaluation after the first CCRT part, ten patients showed CR $(71 \%$ of the included patients). One patient had stable disease and underwent cystectomy. Two patients (14\%) had progressive disease: one underwent cystectomy and the other (who refused radical surgery) continued with the second CCRT part with CR at the end of the treatment. One patient (7\%) could not be evaluated (excluded from the protocol).

The intact bladder preservation rate was $86 \%$ for all included patients (12 out of 14 patients).

\section{Toxicity}

Table 2 reports the acute adverse events that occurred outside the radiation field and could thus be considered as related to chemotherapy. They were mainly hematological disorders, 
digestive alterations and asthenia. Only two of these adverse events were considered DLT (grade 4 thrombocytopenia and grade 3 asthenia). Table 3 reports the adverse events due to radiotherapy in the 14 patients. As expected, urinary discomfort and diarrhea were the two main acute adverse events. No in-field severe events (to be considered as DLT) were observed. Table 4 details the gemcitabine dose, the time of appearance of toxicity, the toxicity grade and DLT leading to chemotherapy delay. DLT was confirmed in two patients. One was a grade 4 thrombocytopenia at day 16 after four injections of $30 \mathrm{mg} / \mathrm{m}^{2}$ gemcitabine and one 4-day continuous infusion of CDDP. Chemotherapy was stopped, and the patient spontaneously recovered. CR was achieved after radiotherapy alone. The second DLT occurred at day 26 and consisted of an overall alteration of the performance status, arrhythmia (auricular fibrillation) and global edema. The patient had already received eight injections of $30 \mathrm{mg} / \mathrm{m}^{2}$ gemcitabine and two 4-day continuous infusions of CDDP. Only radiotherapy was continued and CR was achieved.

\section{Follow-up and survival}

The median follow-up was 53 months (4.4 years) for the whole cohort. Two patients died of progressive metastatic disease. One died of metastatic disease after salvage cystectomy for progressive local disease during the first CCRT part. The second one died of metastatic disease in the first year although the tumor was locally controlled.

Four patients died of undercurrent diseases. One patient died of an ischemic stroke after treatment completion, another of metastatic breast disease and the other two from cardiovascular disease.

The median OS for all patients was 5.8 years. The actuarial OS rates were $79 \%$ (at 2 years), $71 \%$ (3 years) and 62\% (5 years). The median DSS was not reached, but the actuarial DSS 
rate was $77 \%$ at five years. The median BIS was 5.8 years for all patients. The actuarial BIS rates were $64 \%$ at three years and $56 \%$ at five years (Fig.1).

The nine patients who received the whole CCRT protocol had a median OS of 5.8 years, a 5year actuarial DSS rate of $89 \%$ and SIB of $89 \%$ (at three years) and $76 \%$ (five years).

No salvage cystectomy for local tumor recurrence or toxicity was performed during the follow-up period and no significant long-term late toxicity occurred during the follow-up. 


\section{Discussion}

CCRT with CDDP is the most studied protocol for the conservative management of MIBC. Although other systemic drugs (such as 5-FU, taxanes, or gemcitabine) have been already added to CDDP to increase local control, the combination of fractionated pelvic radiotherapy, CDDP and gemcitabine administered twice weekly has never been tested.

We present here the long-term results of our phase I study to assess gemcitabine as a potent radiosensitizer in combination with CDDP for the conservative management of MIBC. A standard 3+3 Phase I dose escalation schedule was planned as no data was available on the twice-weekly gemcitabine and CDDP combination as radiosensitizers. A long-term follow-up of our patients was required as gemcitabine may potentially increase late toxicity, when combined with pelvic radiotherapy. However, the observed toxicity was acceptable with just one grade four thrombocytopenia and one grade four asthenia considered as DLT. No in-field severe event occurred. Regarding the efficacy, our long-term results are quite similar to previously published data in this setting $(18,19)$. Indeed, among patients that received the complete treatment, BIS was $76 \%$ at five years and the median OS was 69.6 months.

Another phase I trial combining gemcitabine and RT with long-term results has been already published, but the pelvic fields were not treated thus making difficult any comparison (20). As usually performed, especially in the trials of the Radiation Therapy Oncology Group (21), we included the pelvic lymph nodes in the radiation field because lymph node involvement is about $20 \%$ and $40 \%$ in pT2 and pT3 tumors, respectively (22). Another phase I study including pelvic fields with gemcitabine reported high toxicity rates and had to be amended to a de-escalation study, with a $25 \mathrm{mg} / \mathrm{m}^{2}$ decrease at each level (23). Consequently, the toxicity observed in this phase I trial should absolutely be interpreted by taking into account this large radiation volume that increases the risk of interactions between ionizing radiation, gemcitabine and CDDP. 
Kent et al. conducted a phase I study on gemcitabine given twice weekly without CDDP and with 60 Gy of radiation after TURBT (17). DLT included deep venous thrombosis, diarrhea, abnormal liver function tests and edema. The recommended dose in this trial was $27 \mathrm{mg} / \mathrm{m}^{2}$ twice-weekly, which is close to the MTD found in our study ( $25 \mathrm{mg} / \mathrm{m}^{2}$ given twice-weekly).

Caffo et al. performed a phase I study on conservative radiotherapy with weekly gemcitabine and $100 \mathrm{mg} / \mathrm{m}^{2}$ CDDP every three weeks (18). Starting with $200 \mathrm{mg} / \mathrm{m}^{2} /$ week gemcitabine, they escalated the dose up to $500 \mathrm{mg} / \mathrm{m}^{2} /$ week, with two DLT: one death and one intestinal perforation. The gemcitabine recommended dose of $400 \mathrm{mg} / \mathrm{m}^{2} /$ week was then evaluated in a phase II study that was unfortunately prematurely closed due to low accrual. The radiotherapy dose was 54 Gy $(1.8 \mathrm{~Gy} /$ fraction) in both trials. A pooled analysis of the two trials on 26 patients was published in 2010 with a median follow-up of 74 months (24). The 5-year clinical outcomes were a 70.1\% OS rate, a 78.9\% DSS rate and a 73.8\% BIS rate. Although this association was effective as all evaluable patients were disease-free at the time of the cystoscopic evaluation, concerns regarding toxicity with the weekly gemcitabine schedule remain. Indeed, intestinal toxicities in the phase I study and neutropenia in the phase II study frequently occurred (even after omission of the gemcitabine injection at day 15 and 36) and for that reason we chose a twice-weekly schedule allowing for lower doses of gemcitabine. In our study, we used standard radiotherapy fractionation (1.8 Gy/fraction/day 5 days a week). However, another group studied gemcitabine radiosensitization with hypofractionated chemoradiotherapy (52.5 Gy in 20 fractions over 28 days) in a phase I study and found a MTD of $100 \mathrm{mg} / \mathrm{m}^{2}$ when delivered only once a week and without CDDP (25). The consecutive phase II trial included 50 patients with transitional cell carcinoma staged T2-3 N0 M0 (19). All patients completed the radiotherapy course; $46(92 \%)$ tolerated all four gemcitabine cycles. Two patients stopped after two cycles, and two after three cycles, due to bowel toxicity. Forty-four (88\%) achieved a complete endoscopic response. At a median follow-up of 36 months (range, 15- 62 months), 36 patients were alive and 32 had a functional and intact 
bladder. By using Kaplan-Meier analyses, the 3-year cancer-specific survival was $82 \%$, and OS was $75 \%$. Nevertheless, these results were obtained with hypofractionated conformal radiotherapy that used a four-field plan with multi-leaf collimators, delivered to the whole bladder and a minimum $1.5-\mathrm{cm}$ margin. Finally, these regimens are clearly manageable for patients with comorbidities but we are still convinced that CDDP presents a strong addedvalue in fit patients.

The results presented here allowed us to determine the gemcitabine MTD when given twice a week in combination with CDDP and radiotherapy. Although bladder preservation and disease control results are promising, this remains a phase I study with only 14 patients and a further validation in a larger cohort is needed. In view of these findings, a French phase II randomized multi-institutional trial named GETUG V04 started in 2011 to assess the efficacy and tolerability of this new combination strategy with gemcitabine twice-weekly compared to the standard CCRT schedule with CDDP alone (NCT01495676). 


\section{References}

1. Dunst J, Sauer R, Schrott KM, et al. Organ-sparing treatment of advanced bladder cancer: a 10-year experience. Int J Radiat Oncol Biol Phys 1994;30:261-266.

2. Tester W, Porter A, Asbell S, et al. Combined modality program with possible organ preservation for invasive bladder carcinoma: results of RTOG protocol 85-12. Int J Radiat Oncol Biol Phys 1993;25:783-790.

3. Kaufman DS, Winter KA, Shipley WU, et al. The initial results in muscle-invading bladder cancer of RTOG 95-06: phase I/II trial of transurethral surgery plus radiation therapy with concurrent cisplatin and 5-fluorouracil followed by selective bladder preservation or cystectomy depending on the initial response. Oncologist 2000;5:471-476.

4. Housset M, Maulard C, Chretien Y, et al. Combined radiation and chemotherapy for invasive transitional-cell carcinoma of the bladder: a prospective study. J Clin Oncol $1993 ; 11: 2150-2157$.

5. Rödel C, Grabenbauer GG, Kühn R, et al. Combined-modality treatment and selective organ preservation in invasive bladder cancer: long-term results. J Clin Oncol 2002;20:3061-3071.

6. Ott OJ, Rödel C, Weiss C, et al. Radiochemotherapy for bladder cancer. Clin Oncol 2009;21:557-565.

7. von der Maase H, Sengelov L, Roberts JT, et al. Long-term survival results of a randomized trial comparing gemcitabine plus cisplatin, with methotrexate, vinblastine, doxorubicin, plus cisplatin in patients with bladder cancer. J Clin Oncol 2005;23:46024608.

8. Kaufman DS, Shipley WU, Feldman AS. Bladder cancer. Lancet 2009;374:239-249.

9. Shewach DS, Lawrence TS. Radiosensitization of human solid tumor cell lines with gemcitabine. Semin Oncol 1996;23:65-71. 
10. Azria $\mathrm{D}$, Jacot $\mathrm{W}$, Prost $\mathrm{P}$, et al. Gemcitabine and ionizing radiations: radiosensitization or radio-chemotherapy combination. Bull Cancer 2002;89:369-379.

11. Sangar VK, Cowan R, Margison GP, et al. An evaluation of gemcitabines differential radiosensitising effect in related bladder cancer cell lines. Br J Cancer 2004;90:542-548.

12. Huguet F, Girard N, Guerche CS-E, et al. Chemoradiotherapy in the management of locally advanced pancreatic carcinoma: a qualitative systematic review. J Clin Oncol 2009;27:2269-2277.

13. Girard N, Mornex F, Douillard J-Y, et al. Is neoadjuvant chemoradiotherapy a feasible strategy for stage IIIA-N2 non-small cell lung cancer? Mature results of the randomized IFCT-0101 phase II trial. Lung Cancer 2010;69:86-93.

14. Shewach DS, Hahn TM, Chang E, et al. Metabolism of 2',2'-difluoro-2'-deoxycytidine and radiation sensitization of human colon carcinoma cells. Cancer Res 1994;54:32183223

15. Lawrence TS, Chang EY, Hahn TM, et al. Radiosensitization of pancreatic cancer cells by 2',2'-difluoro-2'-deoxycytidine. Int J Radiat Oncol Biol Phys 1996;34:867-872.

16. Lawrence TS, Chang EY, Hahn TM, et al. Delayed radiosensitization of human colon carcinoma cells after a brief exposure to 2',2'-difluoro-2'-deoxycytidine (Gemcitabine). Clin Cancer Res 1997;3:777-782.

17. Kent E, Sandler H, Montie J, et al. Combined-Modality Therapy With Gemcitabine and Radiotherapy As a Bladder Preservation Strategy: Results of a Phase I Trial. J Clin Oncol $2004 ; 22: 2540-2545$.

18. Caffo O, Fellin G, Graffer U, et al. Phase I study of gemcitabine and radiotherapy plus cisplatin after transurethral resection as conservative treatment for infiltrating bladder cancer. Int J Radiat Oncol Biol Phys 2003;57:1310-6. 
19. Choudhury A, Swindell R, Logue JP, et al. Phase II Study of Conformal Hypofractionated Radiotherapy With Concurrent Gemcitabine in Muscle-Invasive Bladder Cancer. Journal of Clinical Oncology. 2011;4;29(6):733-8.

20. Oh KS, Soto DE, Smith DC, et al. Combined-Modality Therapy With Gemcitabine and Radiation Therapy as a Bladder Preservation Strategy: Long-Term Results of a Phase I Trial. Int J Radiat Oncol Biol Phys 2009;74:511-517.

21. Mitin T, Hunt D, Shipley WU, et al. Transurethral surgery and twice-daily radiation plus paclitaxel-cisplatin or fluorouracil-cisplatin with selective bladder preservation and adjuvant chemotherapy for patients with muscle invasive bladder cancer (RTOG 0233): a randomised multicentre phase 2 trial. Lancet Oncol 2013;14:863-872.

22. Tarin TV, Power NE, Ehdaie B, et al. Lymph node-positive bladder cancer treated with radical cystectomy and lymphadenectomy: effect of the level of node positivity. Eur Urol 2012;61:1025-1030.

23. Borut K, Lijana ZK. Phase I study of radiochemotherapy with gemcitabine in invasive bladder cancer. Radiother Oncol 2012;102:412-415.

24. Caffo O, Fellin G, Graffer U, et al. Gemcitabine and radiotherapy plus cisplatin after transurethral resection as conservative treatment for infiltrating bladder cancer. Cancer 2011;117:1190-1196.

25. Sangar V, Mcbain C, Lyons J, et al. Phase I study of conformal radiotherapy with concurrent gemcitabine in locally advanced bladder cancer. Int J Radiat Oncol Biol Phys $2005 ; 61: 420-425$. 


\section{Figure legends}

Fig. 1 Survival curves obtained using the Kaplan-Meier method for the overall survival (OS), disease-specific survival (DSS) and bladder-intact survival (BIS). 
Table 1 Patient characteristics

\begin{tabular}{|c|c|}
\hline Characteristic & $\mathrm{n}(\%)$ \\
\hline \\
\hline male & $11(79)$ \\
\hline female & $3(21)$ \\
\hline \multicolumn{2}{|l|}{ Age } \\
\hline median & 72 \\
\hline range & $51-83$ \\
\hline \multicolumn{2}{|l|}{ Histologic grade } \\
\hline poorly differenciated & $10(71)$ \\
\hline moderately differenciated & $3(21)$ \\
\hline unknown & $1(7)$ \\
\hline \multicolumn{2}{|l|}{ TNM stage } \\
\hline $\mathrm{T} 2$ & $12(86)$ \\
\hline T4 & $2(14)$ \\
\hline NO & $14(100)$ \\
\hline MO & $14(100)$ \\
\hline \multicolumn{2}{|l|}{ Gemcitabine biweekly dose $\left(\mathrm{mg} / \mathrm{m}^{2}\right)$} \\
\hline 15 & $3(21)$ \\
\hline 20 & $3(21)$ \\
\hline 25 & $6(43)$ \\
\hline 30 & $2(14)$ \\
\hline
\end{tabular}


Table 2 Acute adverse events occurring outside the radiation fields

\begin{tabular}{lccccc}
\hline Toxicity type & Grade 0, $\mathrm{n}(\%)$ & Grade $1, \mathrm{n}(\%)$ & Grade 2, $\mathrm{n}(\%)$ & Grade 3, $\mathrm{n}(\%)$ & Grade 4, $\mathrm{n}(\%)$ \\
\hline Thrombopenia & $1(7.1)$ & $7(50)$ & $4(28.6)$ & $1(7.1)$ & $1(7.1)$ \\
Neutropenia & $5(35.7)$ & $3(21.4)$ & $2(14.3)$ & $4(28.6)$ & $0(0)$ \\
Anemia & $5(35.7)$ & $7(50)$ & $2(14.3)$ & $0(0)$ & $0(0)$ \\
Infection & $12(85.7)$ & $1(7.1)$ & $1(7.1)$ & $0(0)$ & $0(0)$ \\
Renal & $12(85.7)$ & $1(7.1)$ & $1(7.1)$ & $0(0)$ & $0(0)$ \\
Pulmonary & $12(85.7)$ & $1(7.1)$ & $1(7.1)$ & $0(0)$ & $0(0)$ \\
Fever & $12(85.7)$ & $0(0)$ & $2(14.3)$ & $0(0)$ & $0(0)$ \\
Nausea & $6(42.9)$ & $7(50)$ & $0(0)$ & $1(7.1)$ & $0(0)$ \\
Vomiting & $11(78.6)$ & $1(7.1)$ & $2(14.3)$ & $0(0)$ & $0(0)$ \\
Dyspnea & $13(92.9)$ & $1(7.1)$ & $0(0)$ & $0(0)$ & $0(0)$ \\
Asthenia & $3(21.4)$ & $6(42.9)$ & $4(28.6)$ & $1(7.1)$ & $0(0)$ \\
Insomnia & $13(92.9)$ & $1(7.1)$ & $0(0)$ & $0(0)$ & $0(0)$ \\
Anorexia & $12(85.7)$ & $1(7.1)$ & $1(7.1)$ & $0(0)$ & $0(0)$ \\
Hepatic & $12(85.7)$ & $0(0)$ & $2(14.3)$ & $0(0)$ & $0(0)$ \\
Cutaneous & $13(92.9)$ & $1(7.1)$ & $0(0)$ & $0(0)$ & $0(0)$ \\
Auditive & $14(100)$ & $0(0)$ & $0(0)$ & $0(0)$ & $0(0)$ \\
Neurologic & $14(100)$ & $0(0)$ & $0(0)$ & $0(0)$ & $0(0)$ \\
\hline
\end{tabular}


Table 3 Acute adverse events occurring within the radiation fields

\begin{tabular}{lccccc}
\hline Toxicity type & Grade 0, $\mathrm{n}(\%)$ & Grade 1, $\mathrm{n}(\%)$ & Grade 2, n (\%) & Grade 3, $\mathrm{n}(\%)$ & Grade 4, $\mathrm{n}(\%)$ \\
\hline Urinary incontinence & $11(78.6)$ & $2(15.4)$ & $1(7.1)$ & $0(0)$ & $0(0)$ \\
Urination retention & $13(92.9)$ & $0(0)$ & $1(7.1)$ & $0(0)$ & $0(0)$ \\
Hematuria & $12(85.7)$ & $1(7.1)$ & $0(0)$ & $0(0)$ & $0(0)$ \\
Dysuria & $11(78.6)$ & $3(21.4)$ & $0(0)$ & $0(0)$ & $0(0)$ \\
Pollakiuria & $13(92.9)$ & $0(0)$ & $1(7.1)$ & $0(0)$ & $0(0)$ \\
Diarrhea & $9(64.3)$ & $2(15.4)$ & $3(21.4)$ & $0(0)$ & $0(0)$ \\
Dyserection & $10(90.9)$ & $0(0)$ & $1(9.1)$ & $0(0)$ & $0(0)$ \\
Cutaneous & $13(92.9)$ & $1(7.1)$ & $0(0)$ & $0(0)$ & $0(0)$ \\
\hline
\end{tabular}


Table 4 Toxicity appearance time and dose-limiting toxicity (DLT) confirmation

\begin{tabular}{|c|c|c|c|c|c|}
\hline $\begin{array}{l}\text { Patient } \\
\text { number }\end{array}$ & $\begin{array}{c}\text { Biweekly } \\
\text { gemcitabine } \\
\text { dose }\end{array}$ & $\begin{array}{c}\text { Day since treatment } \\
\text { initiation }\end{array}$ & Toxicity grade & Toxicity type & $\begin{array}{c}\text { Dose-limiting } \\
\text { toxicity }\end{array}$ \\
\hline 1 & 15 & 23 & 3 & neutropenia & No \\
\hline 2 & 15 & 16 & 1 & thrombopenia & No \\
\hline 2 & 15 & 19 & 1 & thrombopenia & No \\
\hline 4 & 20 & 23 & 3 & neutropenia & No \\
\hline 5 & 20 & 16 & 2 & thrombopenia & No \\
\hline 6 & 20 & 12 & 2 & infection & No \\
\hline 6 & 20 & 16 & 2 & infection & No \\
\hline 6 & 20 & 19 & 2 & infection & No \\
\hline 9 & 25 & 12 & 1 & thrombopenia & No \\
\hline 9 & 25 & 16 & 2 & thrombopenia & No \\
\hline 9 & 25 & 19 & 1 & thrombopenia & No \\
\hline 9 & 25 & 33 & 1 & thrombopenia & No \\
\hline 10 & 30 & 16 & 4 & thrombopenia & Yes \\
\hline 11 & 30 & 26 & 3 & asthenia & Yes \\
\hline 12 & 25 & 16 & 3 & thrombopenia & No \\
\hline 12 & 25 & 19 & 2 & thrombopenia & No \\
\hline 12 & 25 & 23 & 1 & thrombopenia & No \\
\hline 12 & 25 & 26 & 1 & thrombopenia & No \\
\hline 12 & 25 & 30 & 1 & thrombopenia & No \\
\hline 12 & 25 & 33 & 1 & thrombopenia & No \\
\hline 13 & 25 & 23 & 2 & neutropenia & No \\
\hline 13 & 25 & 26 & 2 & neutropenia & No \\
\hline 14 & 25 & 16 & 2 & thrombopenia & No \\
\hline 14 & 25 & 19 & 2 & thrombopenia & No \\
\hline
\end{tabular}


Figure 1

Overall Survival
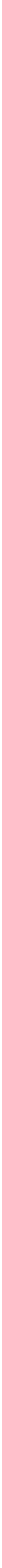
$$
\text { *Uniform Disclosures Form }
$$ \\ Click here to download Uniform Disclosures Form: Azria coi_disclosure.pdf \\ *Uniform Disclosures Form
Click here to download Unife
}


$$
\text { *Uniform Disclosures Form }
$$

*Uniform Disclosures Form
Click here to download Uniform Disclosures Form: Culine coi_disclosure.pdf 
$$
\text { *Uniform Disclosures Form }
$$

Click here to download Uniform Disclosures Form: Fenoglietto coi_disclosure.pdf

*Uniform Disclosures Form
Click here to download Unife 
$$
\text { *Uniform Disclosures Form }
$$

Click here to download Uniform Disclosures Form: Thezenas coi_disclosure.pdf

*Uniform Disclosures Form
Click here to download Unife

lick here to download Uniform Disclosures Form: Thezenas coi_disclosure.pdf 
$$
\text { *Uniform Disclosures Form }
$$

Click here to download Uniform Disclosures Form: Thuret coi_disclosure.pdf

*Uniform Disclosures Form
Click here to download Unife 